\title{
Social Role in Organizational Management Understanding People Behavior and Motivation
}

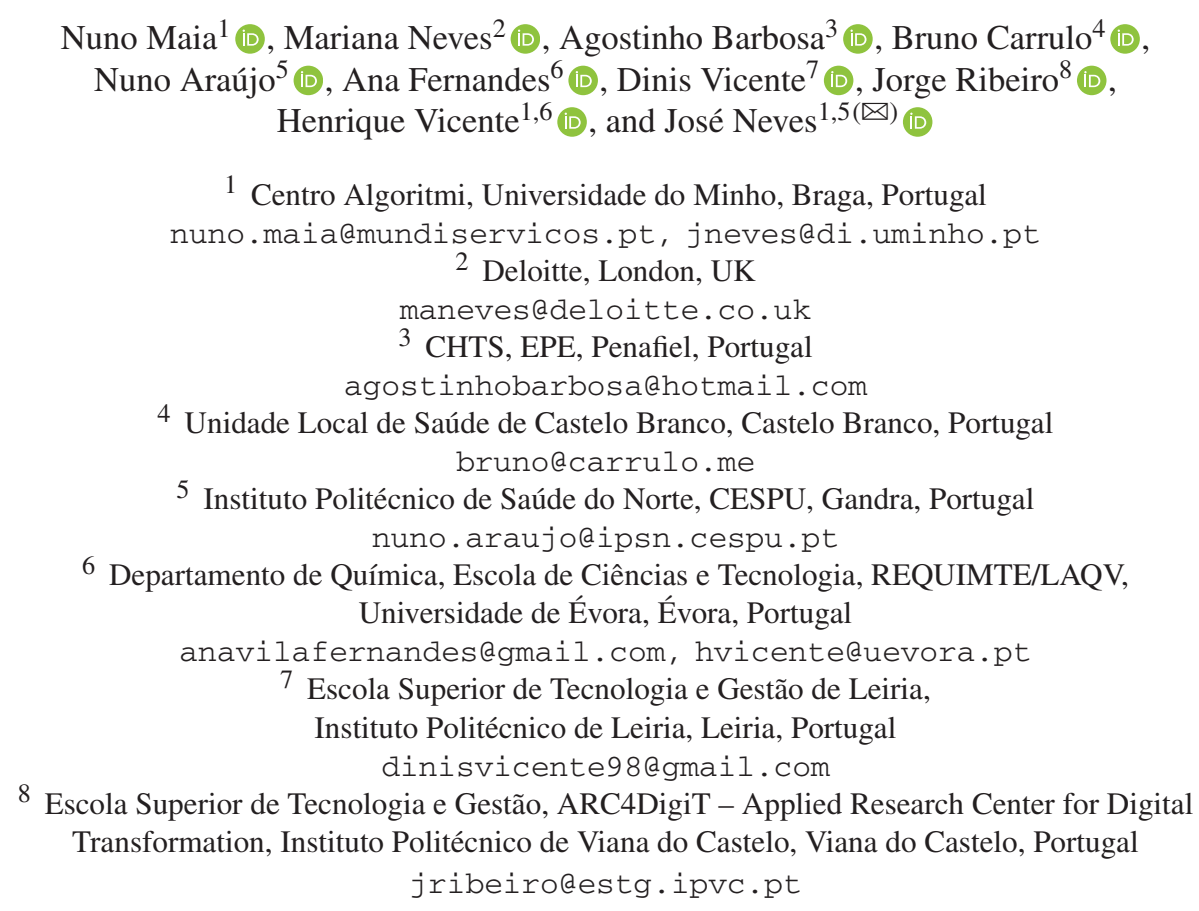

\begin{abstract}
The aim of this work is to respond to the need to rethink the behavior and motivation of employees in their relationship with managers and social groups, i.e., one's main goal is based on increasing engagement in order to reach organizational goals and job workers satisfaction, a complex concept that is influenced by different causes. Indeed, in this work it is analyzed the impact of working conditions on job satisfaction. This is where attention is drawn to the concept of entropy, since we are not focusing on the value a variable can take, but on the effort that has been expended to obtain it. The idea of entropy comes from a principle of thermodynamics dealing with energy. It usually refers to the idea that everything in the universe eventually moves from order to disorder, and entropy is the measurement of that change, that is used here to understand and assess the workers behavior and motivation. The subsequent formal model is based on a set of logical structures for knowledge representation and reasoning that conform to the above entropic view, then leading to an Artificial Neural Network approach to computation, an archetypal that considers the motive behind the action.
\end{abstract}

Keywords: Motivation and Behavior · Job Satisfaction · Entropy $\cdot$ Logic Programming · Knowledge Representation and Reasoning · Artificial Neural Networks 